\title{
Psoriatic epidermis is associated with upregulation of CDK2 and inhibition of CDK4 activity
}

Pauline Henri ${ }^{1}$, Camille Prével ${ }^{1}$, Morgan Pellerano ${ }^{1}$, Julie Lacotte ${ }^{2}$, Pierre-Emmanuel Stoebner ${ }^{1,2}$, May Catherine Morris ${ }^{1}$, Laurent Meunier ${ }^{1,3}$

1 University of Montpellier, IBMM, Montpellier, France, ${ }^{2}$ Dermatology, CHU Nîmes, Nîmes, France, ${ }^{3}$ Dermatology, Hospital St Eloi, Montpellier, France Contact e-mail adress: laurent.meunier@umontpellier.fr

\section{ABSTRACT}

Cyclin-dependent kinases (CDKs) are involved in cell cycle progression. The level of cyclin partners and CDK inhibitors regulate their activity. Psoriasis is a chronic T cell-driven inflammatory skin disease characterized by hyper proliferation of basal epidermal cells. Thanks to fluorescent peptide biosensors, this study demonstrates that epidermal CDK2 activity is increased in psoriatic epidermis while CDK4 activity is completely inhibited. These alterations involve post-translational control mediated by decreased expression of $\mathrm{p} 27^{\text {Kip } 1}(\mathrm{p} 27)$ and $p 16^{\text {INK4A }}(\mathrm{p} 16)$ overexpression, respectively.

\section{OBJECTIVES}

$>$ To monitor CDK4 and CDK2 activity thanks to fluorescent biosensors in human normal and psoriatic epidermis

> To evaluate the expression of Cyclins D1/E and CDK structural inhibitors p16, p21 and p27

1. Induction of CDK4 expression and inhibition of CDK4CycD1 functional activity in human psoriatic epidermis

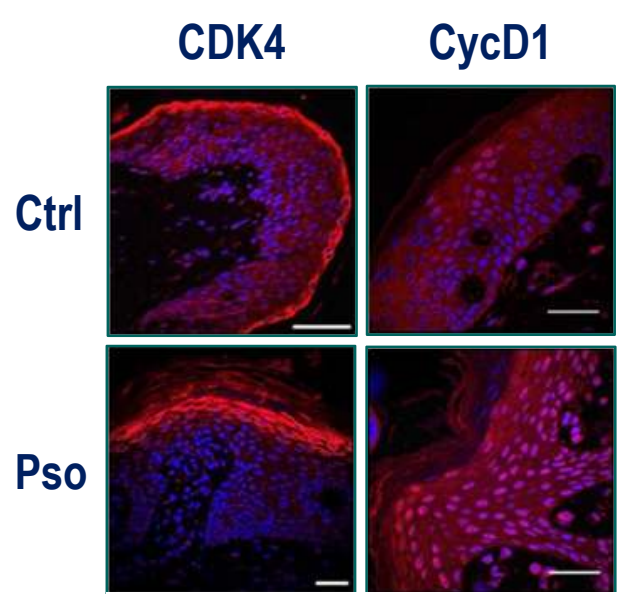

Immunofluorescence stainings showing CDK4 and CycD1 in control and psoriatic skin.

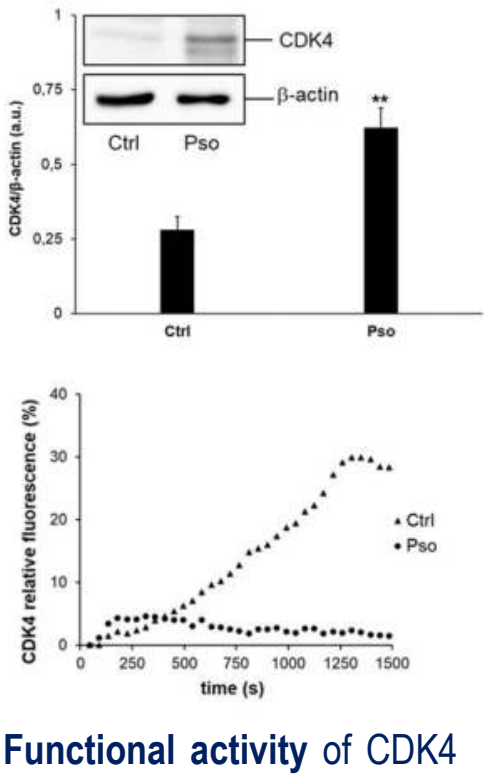
and $\mathrm{CycD} 1$
Western blot analysis of CDK4 in perilesional (Ctrl) and psoriatic (Pso) epidermis.

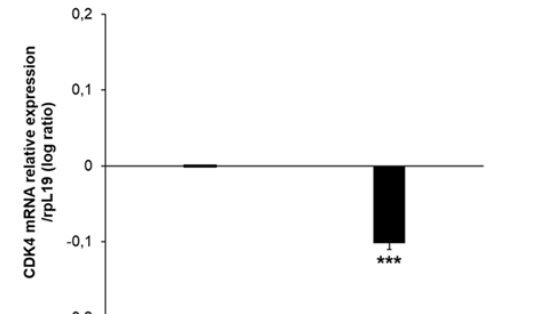

Expression analysis of CDK4 in Ctrl and Pso epidermis using real-time qPCR.
$>$ Both CDK4 and its CycD1 partner are overexpressed in psoriatic epidermis, with CDK4 being principally localized in the cytoplasm and CycD1 accumulating preferentially in the nucleus.

$>$ CDK4 expression was not due to enhanced transcription.

$>$ In contrast there was no increase in CDK4 and CycD1 activity observed, implying that increased levels of these proteins are counteracted by an inhibitory mechanism to restrict kinase function.

\section{METHODOLOGY}

A cohort of 24 patients was asked to participate in the study. Punch biopsies were taken from a chronic plaque and from non-lesional skin of the same patient.

> To monitor CDK2 and CDK4 activity in human epidermis we used CDKACT fluorescent biosensors that undergo fluorescence enhancement upon phosphorylation by CDKs.

> Confocal microscopy, Western blotting and real-time PCR were performed to evaluate expression and transcription of CDK4, CDK2, Cyclins D1/E and CDK inhibitors.
2. Increased expression and activity of the CDK2-CycE complex in psoriatic epidermis

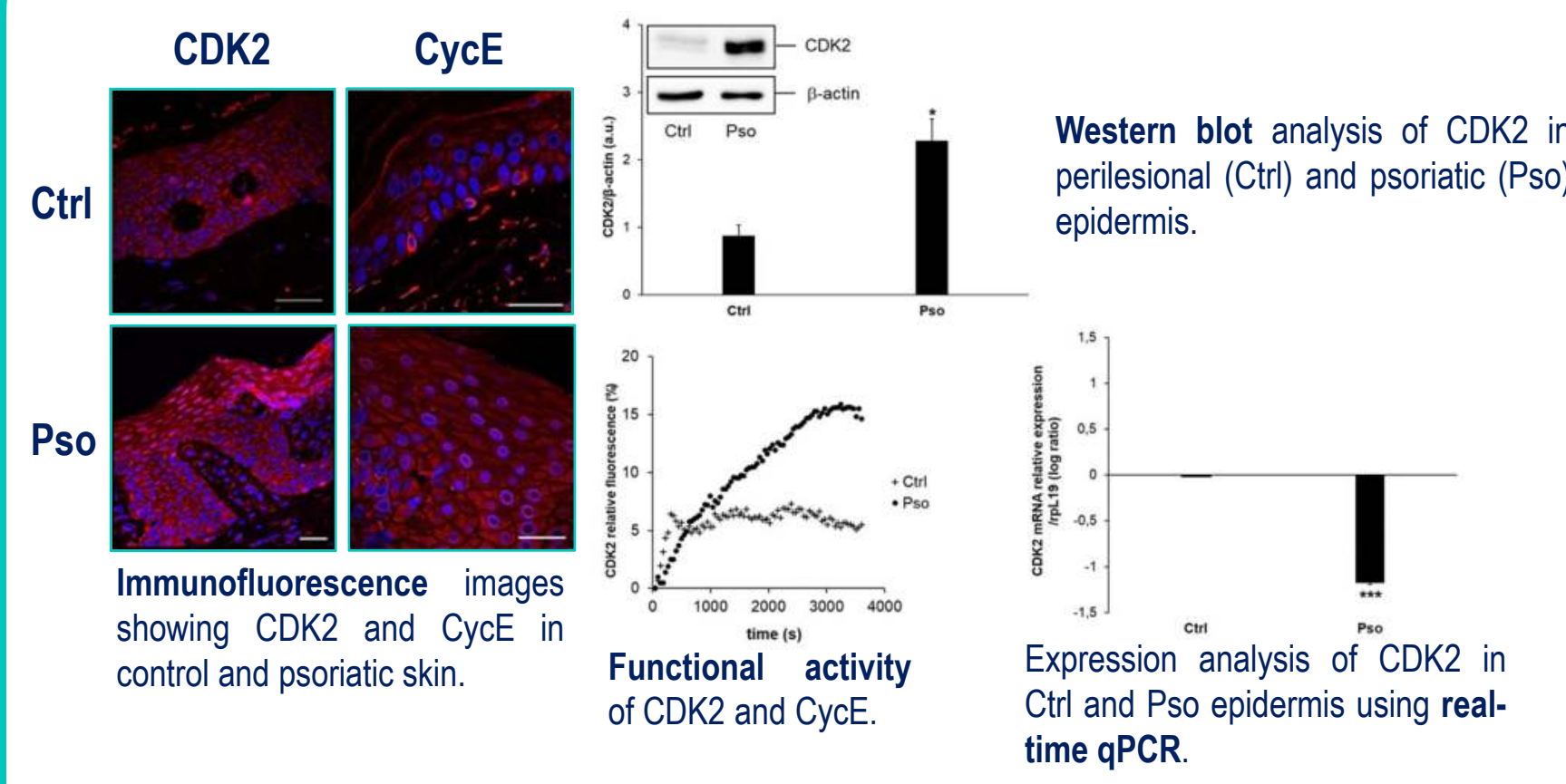

$>$ In psoriatic epidermis both CDK2 and CycE are overexpressed in the nucleus of differentiated epidermal cells.

DEpidermal qPCR analysis demonstrated that increased CDK2 expression was not related to enhanced transcription.

$>$ This overexpression was correlated with a significant increase in CDK2-CycE activity.

\section{Reduced expression of $\mathrm{p} 27$ and induction of the $\mathrm{p} 16$ and $\mathrm{p} 21$ cyclin-dependent kinase inhibitors in psoriatic epidermis}

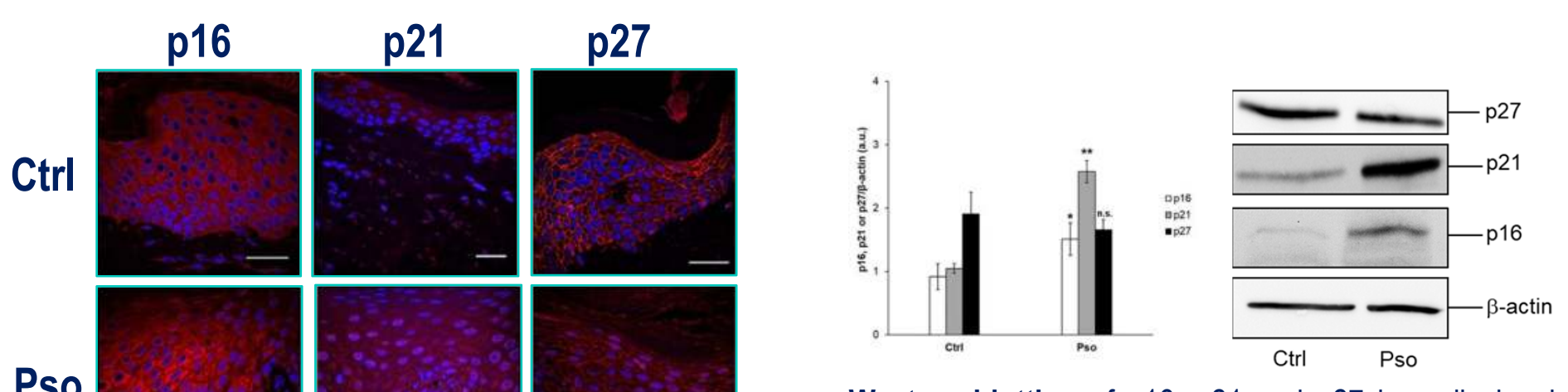

Western blotting of p16, p21 and p27 in perilesional (Ctrl) and psoriatic (Pso) plaques.
$>$ p16 was markedly induced in both the cytoplasm and the nucleus. Western blot analysis confirmed the strong p16 induction in psoriatic skin.

$>$ p21 expression was greatly induced in the cytoplasm and nucleus of psoriatic keratinocytes.

$>$ In normal human skin epidermal p27 expression was strictly restricted to the intercellular spaces, while in psoriatic epidermis, p27 expression was markedly decreased. Western blot analyses of p27 showed no statistically significant difference between psoriatic and control skin.

\section{CONCLUSIONS}

$>$ Epidermal CDK2 activity is increased in psoriatic epidermis while CDK4 activity is completely inhibited. These alterations are not associated with changes in CDK transcription and instead involve post-translational control mediated by decreased expression of p27 and p16 overexpression, respectively.

$>$ Pharmacological modulation of CDK2 and CDK4 may constitute a promising therapeutic strategy. 